\title{
IMU-Camera Self-Calibration Using Planar Mirror Reflection
}

\author{
Ghazaleh Panahandeh and Magnus Jansson \\ KTH Royal Institute of Technology, ACCESS Linnaeus Center, Stockholm, Sweden \\ Email:\{ghazaleh.panahandeh, magnus.jansson\}@ee.kth.se
}

\begin{abstract}
In this paper, we first look at the problem of estimating the transformation between an inertial measurement unit (IMU) and a calibrated camera, based on images of planar mirror reflections (IPMR) of arbitrary feature points with unknown positions. Assuming that only the reflection of the feature points are observable by the camera, the IMUcamera calibration parameters and the position of the feature points in the camera frame are estimated using the Sigma-Point Kalman filter framework. In the next step, we consider the case of estimating varying camera intrinsic parameters using the estimated static parameters from the previous stage. Therefore, the estimated parameters are used as initial values in the state space model of the system to estimate the camera intrinsic parameters together with the rest of the parameters. The proposed method does not rely on using a fixed calibration pattern whose feature points' positions are known relative to the navigation frame. Additionally, the motion of the camera, which is mounted on the IMU, is not limited to be planar with respect to the mirror. Instead, the reflection of the feature points with unknown positions in the camera body frame are tracked over time. Simulation results show subcentimeter and subdegree accuracy for both IMU-camera translation and rotation parameters as well as submillimeter and subpixel accuracy for the position of the feature points and camera intrinsic parameters, respectively.
\end{abstract}

Index Terms-IMU-Camera calibration, IPMR, Sigma-Point Kalman filter, camera intrinsic parameters.

\section{INTRODUCTION}

Inertial navigation systems (INS) use a combination of motion sensors and rotational sensors to provide the position, orientation, and velocity of moving objects. At the core of each INS, there is an inertial measurement unit (IMU) that provides angular velocity and specific force, which yields attitude and position. Due to the integration drift, the position and the attitude must be periodically corrected by input from complementary sensors. One of the most common alternative sensors is radio receivers, such as a global positioning system (GPS) for outdoor positioning. Integration of vision sensors with INS is another alternative that can be used for outdoor as well as indoor positioning. However, aiding the INS with a vision sensor such as a camera requires sensor-to-sensor relative transformation to be known; disregarding such an offset in the system will introduce un-modeled biases that may grow over time. Currently, several in-lab calibration techniques have been proposed to determine the 6 degreesof-freedom (DoF) transformation between the IMU and the camera coordinate frame; however, they are mainly based on 978-1-4577-1804-5/11/\$26.00 (C) 2011 IEEE using a static checkerboard calibration pattern with a known position in the navigation frame.

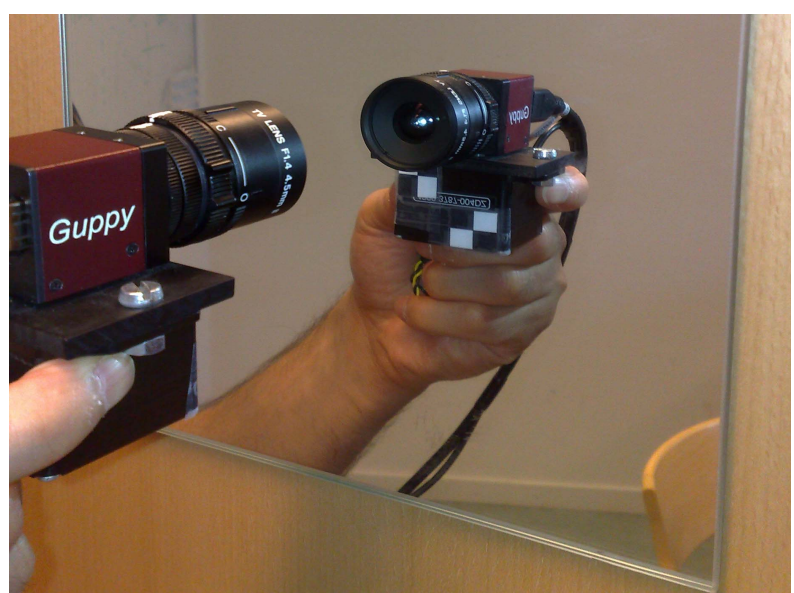

Fig. 1: A monochrome camera rigidly mounted on the top of a IMU, in front of a planar mirror.

For instance, in [1] IMU-camera relative rotation and translation have been estimated separately using gravity as a vertical reference; however, the correlations between the rotation and translation parameters are discarded because of the separated calibration. In [2], calibration parameters are estimated by the extended Kalman filter through tracking static points with known positions in a calibration pattern; furthermore, the observability of the nonlinear system is studied by employing the observability rank condition. A similar nonlinear system is studied by [3], to derive the relative translation and orientation between the IMU and a spherical camera. Using the standard camera calibration pattern, a weighted quadratic cost function has been minimized within the standard gray-box system identification framework.

The main contribution of this paper is to present a novel algorithm for estimating the 6-DoF IMU-camera coordinate transformation via a Sigma-Point Kalman filtering framework [4]; unlike the current calibration approaches, our method does not rely on a direct view of a static calibration pattern with known feature points positions. Arbitrary feature points are selected in the camera body where no prior knowledge is assumed on the pose of the feature points relative to the camera optical center. Then, the calibration procedure tracks virtual views of the arbitrary feature points in a planar mirror. For this reason, the camera along with the IMU is moving in 
front of the planar mirror where the IMU is used to support the camera by providing angular velocity and specific force. Contrary to the existing approach [5], [6], no restriction in the IMU-camera movement is considered except the existence of the feature points in IPMRs. Figure 1 shows an IMU-camera sensor fusion unit in front of a planar mirror.

A planar mirror is a mirror with a planar reflective surface. In this case, an image of an object in front of it appears to be behind the mirror plane. In fact, this image is equivalent to one being captured by a virtual camera that is located behind the mirror; additionally, the position of the virtual camera is symmetric to the position of the real camera. In our case, the reflection of the camera in the planar mirror is used for tracking virtual feature points in the IPMRs.

Using IPMR in [7], the transformation between the camera and the body frame of a robot is determined through the maximum-likelihood estimation framework. However, their method is based on tracking feature points whose position relative to the body frame is assumed to be known in advance. Additionally, it is claimed that no prior knowledge about the robot motion or the mirror configuration is considered; since their method is not based on the epipolar geometry [8], considering such assumptions can be applicable. Considering the epipolar geometry, which relates a pair of images through the fundamental matrix, for the IPMRs is also possible under a certain constraint that limits the camera movement to be planar. In the planar motion, the rotational axis should be normal to the plane containing the direction of translation [5]. As a result the fundamental matrix between IPMRs has 6DoF [8]. For instance in [9], the epipolar geometry of IPMRs is studied to estimate the pose of a real camera moving in front of the mirror. However, the rigid displacements between the virtual views are restricted to be planar.

The above methods all require intrinsically calibrated cameras, which can be achieved using a fixed calibration pattern [10]. However, the availability of the IMU signal is one advantage of such a system that can be addressing the problem of camera self-calibration [11], [12]. For instance, in [11], these parameters are estimated using homographies, on the assumption of known relative rotation from an external sensor. In our previous work [12], the problem of estimating camera intrinsic parameters together with IMU-camera calibration parameters has been studied; however, the algorithm used the assumption of known feature point positions relative to the camera frame.

Due to the camera intrinsic parameter estimation, in the second part of this paper, a camera-self calibration approach is introduced which uses the same IMU-camera and planar mirror structure. Using the estimated IMU-camera calibration parameters and pose of the target feature points in the camera body frame, the camera intrinsic parameters are estimated by IPMRs through the same Sigma-Point Kalman filter framework.

The paper is organized as follows. The process and measurement models, which lead to the state-space equations of the system, is derived in Section II. The structure of the used Sigma-Point Kalman filter algorithm is presented in Section III. The camera intrinsic parameter estimation is studied in Section IV. In Section V, the performance of the proposed method and simulation results in different scenarios are examined. Finally, the conclusion of the study is summarized in Section VI.

In the following sections scalars are denoted by lowercase letters $(s)$, vectors by bold letters $(\mathbf{f})$, and matrices by bold capitals $(\mathbf{K})$.

\section{SySTEM DESCRIPTION}

The goal of our proposed algorithm in this section is to estimate the position and orientation of the camera coordinate frame $\{c\}$ relative to the IMU frame $\{b\}$, where the camera is rigidly mounted in the IMU body frame. In order to simplify the treatment with different coordinate frames, we assume that the navigation frame $\{n\}$ is located in the center of the mirror coordinate frame.

\section{A. Time evolution of the system}

In order to estimate the parameters in the Sigma-Point Kalman filter framework, we first describe the total system state vector as

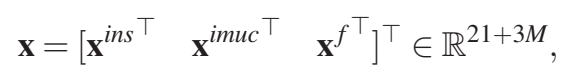

with

$$
\begin{aligned}
& \mathbf{x}^{i n s}=\left[\begin{array}{lllll}
\mathbf{p}_{b}^{n \top} & \mathbf{v}_{b}^{n \top} & \mathbf{q}_{b}^{n \top} & \mathbf{b}_{a}^{\top} & \mathbf{b}_{g}{ }^{\top}
\end{array}\right]^{\top}, \\
& \mathbf{x}^{i m u c}=\left[\begin{array}{ll}
\mathbf{p}_{c}^{b^{\top}} & \mathbf{q}_{c}^{b^{\top}}
\end{array}\right]^{\top} \text {, and } \quad \mathbf{x}^{f}=\left[\pi_{1}^{c \top}, \ldots, \pi_{M}^{c}{ }^{\top}\right]^{\top} .
\end{aligned}
$$

The considered state variables in the INS system are represented by $\mathbf{x}^{i n s}$, where $\mathbf{p}_{b}^{n}$ and $\mathbf{v}_{b}^{n}$ are describing the position and velocity of the IMU in the navigation frame, respectively. $\mathbf{q}_{b}^{n}$ is the unit quaternion representing the rotation from body frame to navigation frame, and $\mathbf{b}_{a}$ and $\mathbf{b}_{g}$ are the bias vectors affecting the accelerometer and gyroscope measurements, respectively. $\mathbf{x}^{\text {iпис }}$ depicts IMU-camera calibration parameters, containing the translation from camera to the body frame $\mathbf{p}_{c}^{b}$, and the unit quaternion representing the rotation from camera frame to body frame $\mathbf{q}_{c}^{b}$. Finally, $\mathbf{x}^{f}$ contains pose of the arbitrary static feature points located in the camera body frame $\left\{\pi_{i}^{c}\right\}_{i=1}^{M} \in \mathbb{R}^{3}$.

The time evolution of the INS state and the IMU-camera transformation [2] can be described by

$$
\begin{aligned}
\dot{\mathbf{q}}_{n}^{b}(t) & =\frac{1}{2} \Omega(\omega(t)) \mathbf{q}_{n}^{b}(t), & \text { where } & \Omega(\omega)=\left[\begin{array}{cc}
-[\omega]_{\times} & \omega \\
-\omega^{\top} & 0
\end{array}\right] \\
\dot{\mathbf{p}}_{b}^{n}(t) & =\mathbf{v}_{b}^{n}(t) & & \dot{\mathbf{v}}_{b}^{n}(t)=\mathbf{a}^{n}(t) \\
\dot{\mathbf{f}}^{b}(t) & =\mathbf{n}_{\delta \mathbf{f}}(t) & \dot{\omega}^{b}(t) & =\mathbf{n}_{\delta \omega}(t) \\
\dot{\mathbf{p}}_{c}^{b}(t) & =\mathbf{0}_{3 \times 1} & \dot{\mathbf{q}}_{c}^{b}(t) & =\mathbf{0}_{3 \times 1}
\end{aligned}
$$

where $\omega(t)$ is the rotational velocity of the body frame and $[\cdot]_{\times}$denotes the skew-symmetric matrix representation of the cross product operation [13]. The IMU and gyroscope bias increments, $\mathbf{n}_{\delta \mathbf{f}}$ and $\mathbf{n}_{\delta \omega}$, are modeled as white Gaussian 
noises. The output measurement signals of the accelerometer $\tilde{\mathbf{f}}_{m}$ and the gyroscope $\tilde{\omega}_{m}$ are modeled as

$$
\begin{aligned}
\tilde{\mathbf{f}}_{m}(t) & =\mathbf{R}_{n}^{b}(t)\left(\mathbf{a}^{n}(t)-\mathbf{g}^{n}\right)+\mathbf{b}_{a}(t)+\mathbf{n}_{\mathbf{f}}(t) \\
\tilde{\omega}_{m}(t) & =\omega(t)+\mathbf{b}_{g}(t)+\mathbf{n}_{\omega}(t)
\end{aligned}
$$

where $\mathbf{R}_{n}^{b}$ is the direction-cosine matrix [13] which can be used as an alternative attitude representation, $\mathbf{n}_{\mathbf{f}}, \mathbf{n}_{\omega}$ are mutually and temporally uncorrelated zero-mean noise processes, and $\mathbf{g}^{n}$ is the gravitational acceleration expressed in the navigation frame.

\section{B. Discrete-time process model}

The discrete-time error state space model is described by

$$
\begin{array}{rlrl}
\delta \mathbf{p}_{b, k+1}^{n} & =\delta \mathbf{p}_{b, k}^{n}+d t \delta \mathbf{v}_{b, k}^{n} & \\
\delta \mathbf{v}_{b, k+1}^{n} & =\delta \mathbf{v}_{b, k}^{n}+d t\left[\hat{\mathbf{R}}_{b, k}^{n} \mathbf{f}_{m, k}\right]_{\times} \delta \theta_{k}+d t \hat{\mathbf{R}}_{b, k}^{n}\left(\delta \mathbf{f}_{k}^{b}+\mathbf{n}_{\mathbf{f}, k}\right) \\
\delta \theta_{k+1} & =\delta \theta_{k}-d t \hat{\mathbf{R}}_{b, k}^{n}\left(\delta \omega_{k}^{b}+\mathbf{n}_{\omega, k}\right) \\
\delta \mathbf{f}_{k+1}^{b} & =\delta \mathbf{f}_{k}^{b}+d t \mathbf{n}_{\delta \mathbf{f}, k} & \delta \omega_{k+1}^{b}=\delta \omega_{k}^{b}+d t \mathbf{n}_{\delta \omega, k} \\
\delta \mathbf{x}_{k+1}^{i m u c} & =\delta \mathbf{x}_{k}^{\text {imuc }} & \delta \mathbf{x}_{k+1}^{f}=\delta \mathbf{x}_{k}^{f}
\end{array}
$$

where $\hat{\mathbf{R}}_{b}^{n}$ is the estimated rotation matrix which can be estimated using the measurement and INS equation (3) and (2), respectively. The equations in (4) have been derived based on the standard additive error definition for the position, velocity, and biases $(\hat{x} \simeq x+\delta x)$ and quaternion error for the rotational angles $\psi\left(\delta \mathbf{q} \simeq\left[\begin{array}{ll}1 & \frac{\delta \psi}{2}\end{array}\right]^{\top}\right)$; the advantage of quaternion error definition is the direct use of error angle vectors $\delta \theta$ and $\delta \varphi$ for the $\mathbf{q}_{b}^{n}$ and $\mathbf{q}_{c}^{b}$, respectively.

According to the state vector model (1) and the discretetime error state space model (4), the total error state vector is written as

$$
\delta \mathbf{x}=\left[\begin{array}{lll}
\delta \mathbf{x}^{i n s^{\top}} & \delta \mathbf{x}^{i m u c}{ }^{\top} \quad \delta \mathbf{x}^{f^{\top}}
\end{array}\right]^{\top}
$$

with

$$
\begin{aligned}
& \delta \mathbf{x}^{i n s}=\left[\begin{array}{lllll}
\delta \mathbf{p}_{b}^{n \top} & \delta \mathbf{v}_{b}^{n \top} & \delta \theta^{\top} & \delta \mathbf{b}_{a}{ }^{\top} & \delta \mathbf{b}_{g}^{\top}
\end{array}\right]^{\top}, \\
& \delta \mathbf{x}^{i m u c}=\left[\begin{array}{ll}
\delta \mathbf{p}_{c}^{b^{\top}} & \delta \varphi^{\top}
\end{array}\right]^{\top} \text {, and } \delta \mathbf{x}^{f}=\left[\delta \pi_{1}^{c \top}, \ldots, \delta \pi_{M}^{c}{ }^{\top}\right]^{\top} \text {. }
\end{aligned}
$$

Hence, the state-space model of the discrete time process is given by

$$
\delta \mathbf{x}_{k+1}=f_{k}\left(\delta \mathbf{x}_{k}, \mathbf{n}_{k}\right) \in \mathbb{R}^{21+3 M}
$$

where the process noise $\mathbf{n}_{k}=\left[\begin{array}{llll}\mathbf{n}_{\mathbf{f}, k}^{\top} & \mathbf{n}_{\omega, k}^{\top} & \mathbf{n}_{\delta \mathbf{f}, k}^{\top} & \mathbf{n}_{\delta \omega, k}^{\top}\end{array}\right]^{\top}$ is assumed to be time invariant, with the covariance matrix $\mathbf{Q} \in$ $\mathbb{R}^{12 \times 12}$.

\section{Mirror reflection transformation}

To define the discrete time measurement model of the system, first of all, we need to derive the geometry of the reflected point in the planar mirror. Without loss of generality, a standard right-handed Cartesian coordinate system is used for all the coordinate frames. The navigation frame, $\{n\}$, is located in the center of the mirror coordinate frame (see Figure 2) such that its $x y$ plane is the reflective surface. For the sake of simplicity, equations are derived for only one feature

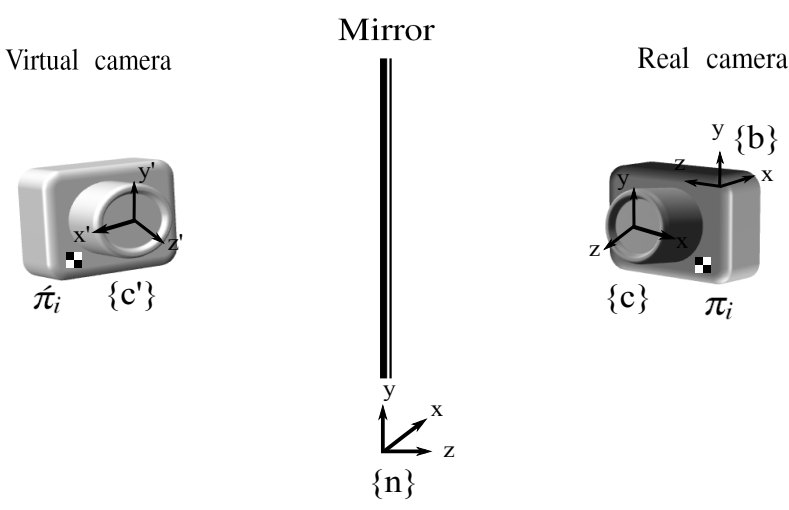

Fig. 2: System sensor structure in front of the planar mirror

point $\pi_{i}$ which is selected arbitrary in the camera body frame. Denoting the reflection of $\pi_{i}$ in the planar mirror by $\tilde{\pi}_{i}$, they can be related in the navigation frame via the reflection matrix A as

$$
\tilde{\pi}_{i}^{n}=\mathbf{A} \pi_{i}^{n} \in \mathbb{R}^{3}, \quad \text { where } \quad \mathbf{A}=\left[\begin{array}{ccc}
1 & 0 & 0 \\
0 & 1 & 0 \\
0 & 0 & -1
\end{array}\right] .
$$

The geometric relation between the pose of $\pi_{i}$ and $\tilde{\pi}_{i}$ can also be described in the camera coordinate frame by considering the trasformation between the camera and the navigation frame as

$$
\begin{aligned}
\pi_{i}^{c} & =\mathbf{R}_{n}^{c} \pi_{i}^{n}+\mathbf{p}_{n}^{c} \\
\tilde{\pi}_{i}^{c} & =\mathbf{R}_{n}^{c} \hat{\pi}_{i}^{n}+\mathbf{p}_{n}^{c} .
\end{aligned}
$$

By substituting (8a) and (7) into (8b), we have

$$
\hat{\pi}_{i}^{c}=\mathbf{R}_{n}^{c} \mathbf{A R}_{n}^{c^{\top}}\left(\pi_{i}^{c}-\mathbf{p}_{n}^{c}\right)+\mathbf{p}_{n}^{c} .
$$

Inserting the IMU-camera transformation, $\mathbf{R}_{n}^{c}=\mathbf{R}_{c}^{b^{\top}} \mathbf{R}_{n}^{b}$ and $\mathbf{p}_{c}^{n}=\mathbf{p}_{b}^{n}+\mathbf{R}_{n}^{b^{\top}} \mathbf{p}_{c}^{b}$, in (9) and using the fact that $\mathbf{p}_{c}^{n}=-\mathbf{R}_{n}^{c \top} \mathbf{p}_{n}^{c}$, the vector $\hat{\pi}_{i}^{c}$ can be rewritten as

$$
\begin{aligned}
\tilde{\pi}_{i}^{c}= & \mathbf{R}_{c}^{b^{\top}} \mathbf{R}_{n}^{b} \mathbf{A}\left(\mathbf{R}_{c}^{b^{\top}} \mathbf{R}_{n}^{b}\right)^{\top} \pi_{i}^{c}-\mathbf{R}_{c}^{b^{\top}} \mathbf{R}_{n}^{b}\left(\mathbf{I}_{3}-\mathbf{A}\right) \mathbf{p}_{b}^{n} \\
& -\left(\mathbf{I}_{3}-\mathbf{R}_{c}^{b^{\top}} \mathbf{R}_{n}^{b} \mathbf{A}\left(\mathbf{R}_{c}^{b^{\top}} \mathbf{R}_{n}^{b}\right)^{\top}\right) \mathbf{R}_{c}^{b^{\top}} \mathbf{p}_{c}^{b} .
\end{aligned}
$$

Using the unit vector along the $z$ axis, $\mathbf{e}_{z}=[0,0,1]^{\top}$, the matrix A can be decomposed as $\mathbf{A}=\mathbf{I}_{3}-2 \mathbf{e}_{z} \mathbf{e}_{z}^{\top}$, where $\mathbf{I}_{3}$ is the identity matrix. By replacing $\mathbf{A},(10)$ can be simplified to

$$
\tilde{\pi}_{i}^{c}=\pi_{i}^{c}-2 \mathbf{R}_{c}^{b^{\top}} \mathbf{R}_{n}^{b} \mathbf{e}_{z} \mathbf{e}_{z}^{\top}\left(\mathbf{R}_{n}^{b^{\top}} \mathbf{R}_{c}^{b} \pi_{i}^{c}+\mathbf{p}_{b}^{n}+\mathbf{R}_{n}^{b^{\top}} \mathbf{p}_{c}^{b}\right) .
$$

Finally, the position of the reflected feature points in the camera coordinate frame, which will be used in the camera projection model in Section II-D, is described as a function of our defined states (1). The main advantage of this model is that instead of using $\hat{\pi}_{i}^{c}$ that maybe change from image to image while the camera is moving, $\pi_{i}^{c}$ is used which is fixed in the camera body frame and can be estimated efficiently over time by the Kalman filter framework. This implies, although no prior knowledge about the pose of $\pi_{i}$ is assumed, it should be static relative to the camera and visible in each captured image. 


\section{Measurement model}

When the camera along with the IMU is moving in front of a planar mirror, the body frame angular velocity and specific force are measured by the IMU. Meanwhile, the camera records IPMRs. The projection of the reflected feature point $\tilde{\pi}_{i}^{c}$ to the image plane, based on the pinhole camera model [8], can be represented by

$$
\mathbf{z}_{i}=h_{i}\left(\mathbf{x}^{i n s}, \mathbf{x}^{i m u c}, \dot{\pi}_{i}^{c}\right)+\mathbf{v}_{i}=\left[\begin{array}{ll}
\mathbf{I}_{2} & \mathbf{0}
\end{array}\right] \mathbf{K}\left[\begin{array}{c}
\hat{\pi}_{i, x}^{c} \\
\dot{\pi}_{i, y}^{c} \\
\hat{\pi}_{i, z}^{c}
\end{array}\right] \frac{1}{\hat{\pi}_{i, z}^{c}}+\mathbf{v}_{i} \in \mathbb{R}^{2}
$$

where $\mathbf{v}_{i}$ is the feature-measurement noise with covariance matrix $\mathbf{R}_{i}=\sigma_{v}^{2} \mathbf{I}_{2}$, and $\mathbf{K}$ is the camera intrinsic matrix, assumed to be known. It can be represented by

$$
\mathbf{K}=\left[\begin{array}{ccc}
k_{u} & s & p_{u} \\
0 & k_{v} & p_{v} \\
0 & 0 & 1
\end{array}\right]
$$

where $k_{u}$ and $k_{v}$ are the magnifications in the two coordinate directions of the image, $s$ is a skew parameter corresponding to a skewing of the coordinate axes, and $p_{u}$ and $p_{v}$ are the coordinates of the principal point [8].

For $M$ observed reflected feature points described by (11), the discrete-time measurement model of the system at the $k$ th time instant is

$$
\delta \overline{\mathbf{z}}_{k}=h_{k}\left(\delta \mathbf{x}_{k}^{i n s}, \delta \mathbf{x}_{k}^{i m u c}, \delta \mathbf{x}_{k}^{f}\right)+\overline{\mathbf{v}}_{k} \in \mathbb{R}^{2 M} .
$$

Assuming the measurement noise from each projection of the reflected feature points to be mutually uncorrelated, the covariance matrix of $\overline{\mathbf{v}}_{k}, \overline{\mathbf{R}}^{2 M \times 2 M}$, will be block-diagonal.

\section{Sigma-Point Kalman Filtering}

Using the Sigma-Point Kalman Filter framework [4], the statistics of the random variables under the nonlinear process model (6) and measurement model (13) are calculated. Additionally, this technique alleviates the requirement to explicitly calculate Jacobians, which for complex functions, such as (11), can be a difficult task or numerically unstable. To ensure numerical stability, the filter can be implemented in square-root form [14]. By propagating the square root of the covariance matrix there is no need to factorize the covariance matrix by Cholesky decomposition in the Sigma-Point Kalman filter; furthermore, using the triangular matrix square-root of the covariance matrix ensures the positive definiteness.

The high-rate IMU signal measurement propagates the state and the covariance matrix before a new measurement is received. From each new captured image the reflection of feature points are detected, then the state estimates and the covariance matrices of the system are updated.

An overview of the algorithm is given in Algorithm 1, where $\chi_{l}$ is the $l$-th column of the Sigma-Points matrix $\chi$, $N$ is the length of the state vector, and nd $\mathbf{G}$ is the system noise propagation matrix. The weights of the Sigma-Point filter $w_{l}^{c}, w_{l}^{m}$ are set to $\frac{1}{2(N+1)}$ for $l=1, \cdots, 2 N$, and for $l=0$ $w_{0}^{c}=\frac{\lambda}{N+\lambda}+\left(1-\alpha^{2}+\beta\right), w_{0}^{m}=\frac{\lambda}{N+\lambda}$. The scaling parameter
$\lambda=\alpha^{2}(N+\kappa)-N$, and $\eta=\sqrt{N+\lambda}$ are set with $\alpha=0.1$, $\beta=2$, and $\kappa=0$.

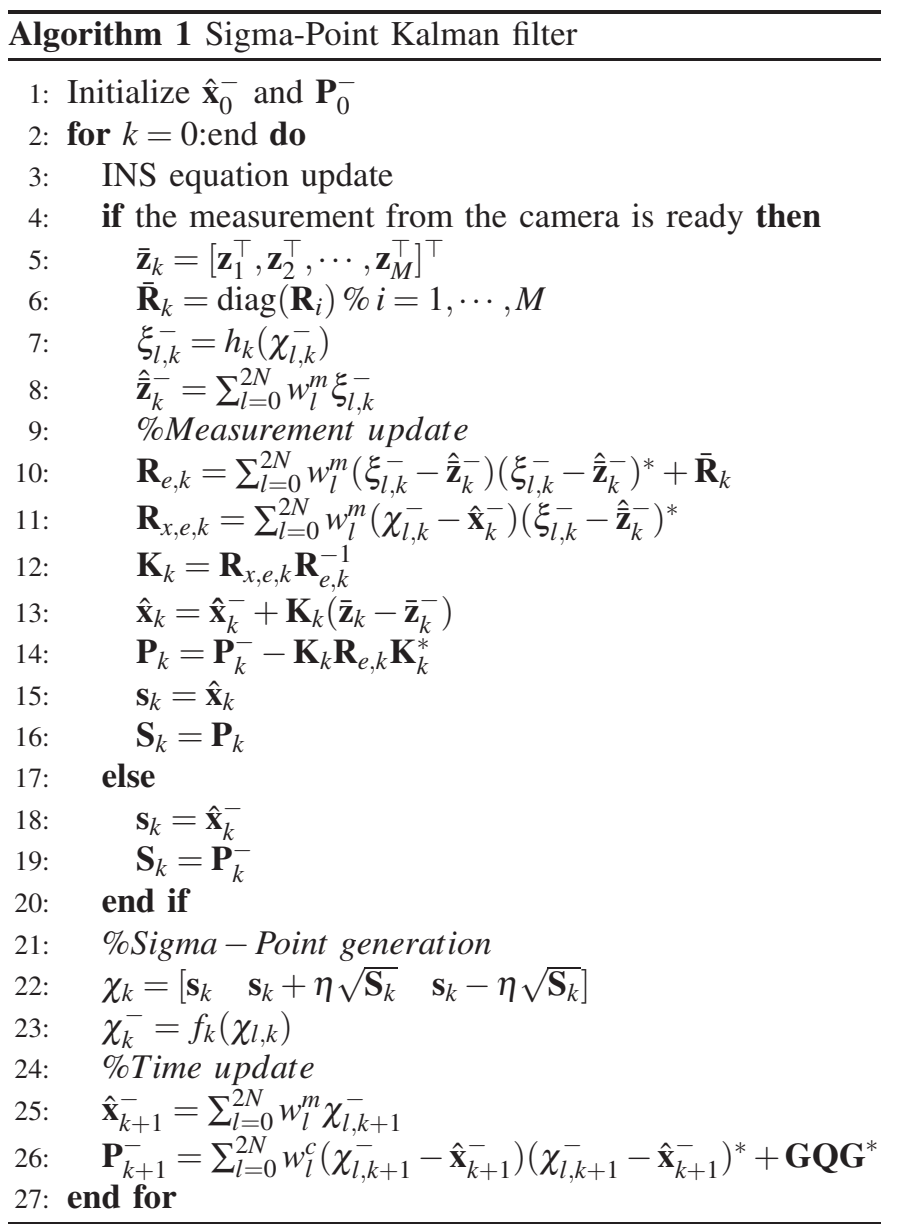

\section{CAMERA INTRINSIC PARAMETER ESTIMATION}

The proposed approach in Section III provides an accurate estimate of the static IMU-camera calibration parameters and the positions of the feature points for a calibrated camera. However, in many scenarios the camera intrinsic parameters may change during the measurement (e.g, the focal length). Moreover, there are cases in which the camera can be replaced with a new vision sensor in the same sensor fusion configuration.

In this section, the previous system structure is extended to estimate the varying camera intrinsic parameters using the estimated IMU-camera transition and feature points positions as an initial value in the same Sigma-Point Kalman filter framework. The proposed algorithms for IMU-camera sensor fusion provide, first, the ability of estimating IMU-camera calibration parameters for any camera with known intrinsic parameters, secondly, the opportunity of estimating the camera intrinsic parameters for the IMU-camera system which has been calibrated within the first stage.

Concatenating the camera intrinsic parameters (12) as $\mathbf{x}^{c c}=$ $\left[\begin{array}{lllll}k_{u} & k_{v} & p_{u} & p_{v} & s\end{array}\right]^{\top}$ to the system state vector (1), the 


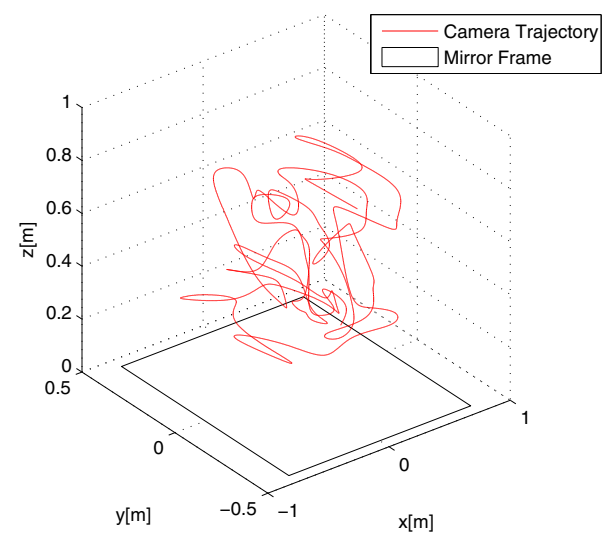

Fig. 3: IMU trajectory over a planar mirror, for 12 seconds.

extended system state vector can be written as

$$
\mathbf{x}=\left[\begin{array}{lll}
\mathbf{x}^{i n s} \mathbf{x}^{\text {imuc }}{ }^{\top} & \mathbf{x}^{f^{\top}} & \mathbf{x}^{c c \top}
\end{array}\right]^{\top} \in \mathbb{R}^{26+3 M} .
$$

Considering $\delta \mathbf{x}_{k+1}^{c c}=\delta \mathbf{x}_{k}^{c c}$ in the discrete time error state space model, the total error state vector is expanded to

$$
\delta \mathbf{x}=\left[\begin{array}{lll}
\delta \mathbf{x}^{i n s}{ }^{\top} & \delta \mathbf{x}^{i m u c}{ }^{\top} \quad \delta \mathbf{x}^{f^{\top}} \quad \delta \mathbf{x}^{c c \top}
\end{array}\right]^{\top} .
$$

Hence, the discrete-time measurement model of the system (13) will be a function of $\delta \mathbf{x}^{c c}$ as well as the rest of the parameters (5). Algorithm 1 can be used for estimating the parameters of the extended state vector (14), the only difference is the number of unknown parameters $N$ in the state vector which must be changed to $26+2 M$.

\section{Performance Evaluation}

The proposed calibration approach has been evaluated by Monte-Carlo simulations. The example IMU movement trajectory which is used for the performance evaluation of the estimators is shown in Figure 3. It displays a movement with roll $\phi \in\left[-90^{\circ}, 90^{\circ}\right]$ in a period of 12 seconds with a peak speed of about $0.4[\mathrm{~m} / \mathrm{s}]$ and angular rate of 2.9 [rad./s].

The sampling rate of the accelerometer and gyroscope output signals, $\mathbf{f}_{k}^{b}$ and $\omega_{k}^{b}$, is $100[\mathrm{~Hz}]$. The IMU accelerometers and gyros biases are set to $2 \cdot 10^{-3}\left[\mathrm{~m} / \mathrm{s}^{2}\right]$ and $5 \cdot 10^{-4}$ [rad./s] with the standard deviation of $6 \cdot 10^{-3}$ and $3 \cdot 10^{-3}$, respectively.

The camera sampling rate is $10[\mathrm{~Hz}]$ and its intrinsic parameters are set to $k_{u}=k_{v}=833, p_{u}=2, p_{v}=8$, and $s=3$. The IMU-camera translation is $\mathbf{p}_{c}^{b}=[1,-5,10]^{\top}[\mathrm{cm}]$ and the rotation is set to $\mathbf{R}_{c}^{b}=\left[-90^{\circ}, 0^{\circ},-90^{\circ}\right]$. Our estimation method has been evaluated using three feature points placed in the camera body frame. In fact, at least three non-collinear points are necessary to be selected in the camera frame to define the camera coordinate frame [7].

\section{A. Filter Initialization}

The initial value for the 6-DoF IMU-camera transformation can be computed manually from the computer aided design (CAD) plots, or by using the static measurement of the gravitational field [1], [15]. In our simulation, the initial values are set by adding a random noise from a Gaussian distribution with zero mean and standard deviation of $\sigma$ to the true values.

To estimate the IMU-camera calibration parameters and pose of the selected feature points in the camera body frame, which we are really interested to estimate, along with the rest of the state variables $\mathbf{x}^{i n s}$, which are referred to as nuisance parameters, the initial value of the error state estimates $\delta \hat{\mathbf{x}}$ are set to be zero. The standard deviations of the initial estimation error of the IMU position and the IMU-camera translation is set to $1 \cdot 10^{-2}[\mathrm{~m}]$ and for the velocity $1 \cdot 10^{-2}[\mathrm{~m} / \mathrm{s}]$. The standard deviations for the bias error estimates are set to $4 \cdot 10^{-2}\left[\mathrm{~m} / \mathrm{s}^{2}\right]$ and $1 \cdot 10^{-3}[\mathrm{rad} / \mathrm{s}]$, respectively. The value of the error state estimates in the attitude and IMU-camera rotation is initialized with $2\left[^{\circ}\right]$.

In order to initialize the filter for estimating camera intrinsic parameters, the final estimates of the unknown parameters (1) and their corresponding uncertainties in the first stage are used in the second stage of the proposed approach. The initial standard deviation of the camera intrinsic parameters are set to 100 [pixel] .

To form $\hat{\mathbf{x}}_{0}^{-}$, actual initial errors were generated by a zeromean Gaussian distribution with corresponding covariance matrix in both stages. The diagonal error covariance matrix $\mathbf{P}_{0}^{-}$was initialized by the corresponding standard deviations.

\section{B. Simulation Results}

The estimated mean errors along with the root mean square error (RMSE) and $3 \sigma$-levels for the IMU-camera transformation and one of the feature point's position in the camera body frame are plotted in Figure 4-5 over 12 seconds, using 100 Monte-Carlo simulations. The $3 \sigma$-levels are computed from the corresponding diagonal elements of the filter's error covariance matrix that provides a representation of its estimate uncertainty. Zero-mean Gaussian noise with standard deviation of $\sigma_{v}=2$ [pixel] is added to the projected image.

Table I and II summarize the final estimated values and the standard deviation of the error $(\sigma)$ for IMU-camera 6-DoF, translation and rotation, and the position of feature points. Simulation results show that the proposed estimation method is able to reach subcentimeter and subdegree accuracy for the IMU-camera rotation and translation as well as subcentimeter errors for the position of feature points on the camera body frame.

Furthermore, the estimated mean errors along with the root mean square error (RMSE) and $3 \sigma$-levels for the camera intrinsic parameters are plotted in Figure 6 as the result of the second stage of the proposed algorithm, similarly, over 12 seconds, using 100 Monte-Carlo simulations, and $\sigma_{v}=2$. The initial and final estimate of camera intrinsic parameters using the estimated value of the IMU-camera 6-DoF and feature point positions as the initial values in the extended SigmaPoint Kalman filter are shown in Table III.

\section{CONCLUSION}

An approach for IMU-camera self-calibration has been proposed for estimating the 6-DoF IMU-camera transforma- 
TABLE II: Initial, final, and error statistics of 3 selected feature points positions, for 100 Monte Carlo simulations and calibrated camera.

\begin{tabular}{|c|c|c|c||c|c|c||c|c|c|}
\cline { 2 - 10 } \multicolumn{1}{c|}{} & $\pi_{x, 1}^{c} \pm \sigma[\mathrm{cm}]$ & $\pi_{y, 1}^{c} \pm \sigma[\mathrm{cm}]$ & $\pi_{z, 1}^{c} \pm \sigma[\mathrm{cm}]$ & $\pi_{x, 2}^{c} \pm \sigma[\mathrm{cm}]$ & $\pi_{y, 2}^{c} \pm \sigma[\mathrm{cm}]$ & $\pi_{z, 2}^{c} \pm \sigma[\mathrm{cm}]$ & $\pi_{x, 3}^{c} \pm \sigma[\mathrm{cm}]$ & $\pi_{y, 3}^{c} \pm \sigma[\mathrm{cm}]$ & $\pi_{z, 3}^{c} \pm \sigma[\mathrm{cm}]$ \\
\hline Initial & $2 \pm 10$ & $-3 \pm 10$ & $-3 \pm 10$ & $5 \pm 10$ & $4 \pm 10$ & $-3 \pm 10$ & $-8 \pm 10$ & $6 \pm 10$ & $1 \pm 10$ \\
\hline Final & $1.98 \pm 0.03$ & $-2.99 \pm 0.03$ & $-3.00 \pm 0.03$ & $4.98 \pm 0.05$ & $4.00 \pm 0.04$ & $-3.00 \pm 0.03$ & $-8.01 \pm 0.08$ & $6.00 \pm 0.06$ & $0.99 \pm 0.01$ \\
\hline
\end{tabular}
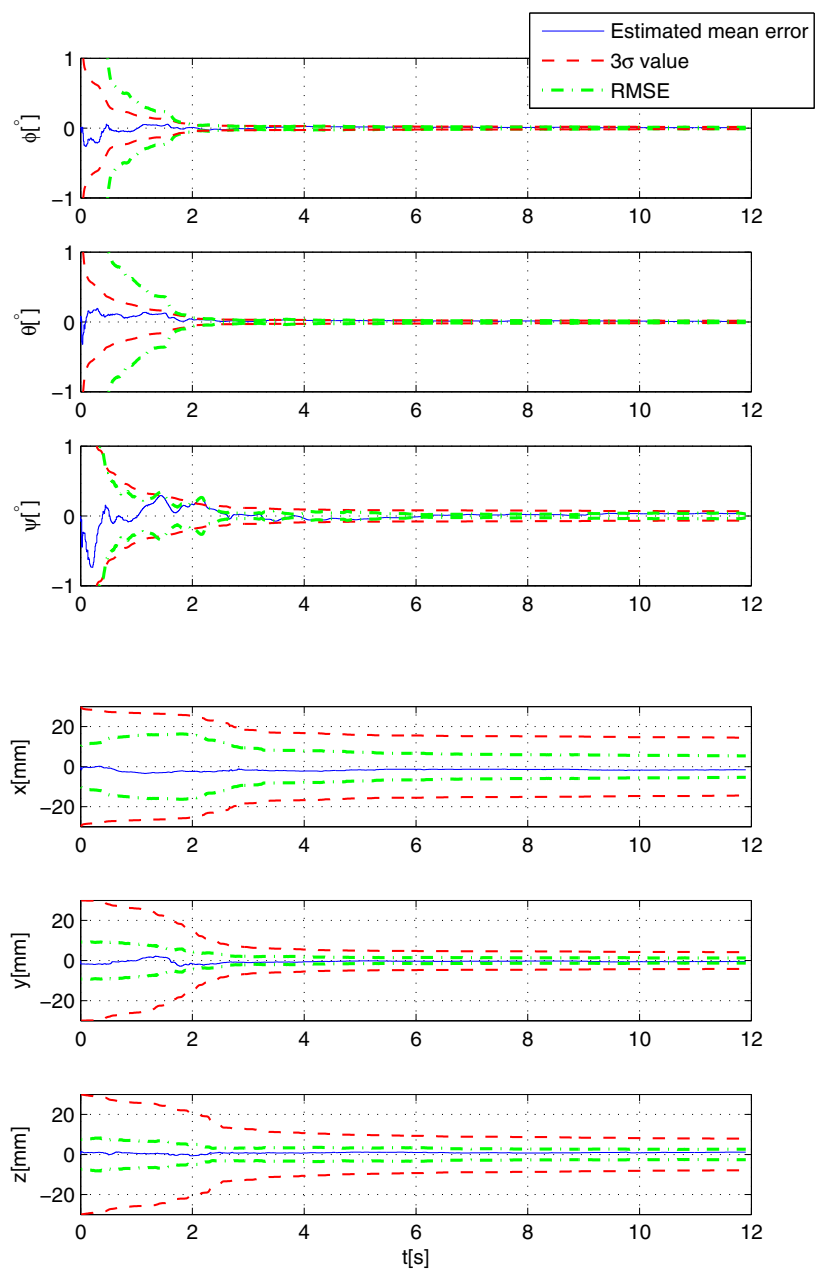

Fig. 4: Estimation error of the IMU-camera transformation.

TABLE I: Initial, final, and error statistics of the IMU-camera transformation, for 100 Monte Carlo simulations and calibrated camera.

\begin{tabular}{|c|c|c|c||c|c|c|}
\cline { 2 - 7 } \multicolumn{1}{c|}{} & $\mathbf{p}_{c, x}^{b} \pm \sigma[\mathrm{cm}]$ & $\mathbf{p}_{c, y}^{b} \pm \sigma[\mathrm{cm}]$ & $\mathbf{p}_{c, z}^{b} \pm \sigma[\mathrm{cm}]$ & $\varphi_{\phi} \pm \sigma\left[^{[0}\right]$ & $\varphi_{\theta} \pm \sigma\left[^{0}\right]$ & $\varphi_{\psi} \pm \sigma\left[^{\circ}\right]$ \\
\hline Initial & $1 \pm 1$ & $-5 \pm 1$ & $10 \pm 1$ & $-90 \pm 2$ & $0 \pm 2$ & $-90 \pm 2$ \\
\hline Final & $0.69 \pm 0.53$ & $-5.08 \pm 0.16$ & $10.12 \pm 0.31$ & $-89.99 \pm 0.01$ & $0 \pm 0.02$ & $-89.98 \pm 0.06$ \\
\hline
\end{tabular}

TABLE III: Initial, final, and error statistics of the camera intrinsic parameters, for 100 Monte Carlo simulations.

\begin{tabular}{|c|c|c|c|c|c|}
\cline { 2 - 6 } \multicolumn{1}{c|}{} & $k_{u} \pm \sigma$ [pixel] & $k_{v} \pm \sigma$ [pixel] & $p_{u} \pm \sigma$ [pixel] & $p_{v} \pm \sigma$ [pixel] & $s \pm \sigma[$ pixel] \\
\hline Initial & $833.33 \pm 100$ & $833.33 \pm 100$ & $2 \pm 100$ & $8 \pm 100$ & $3 \pm 100$ \\
\hline Final & $833.93 \pm 1.26$ & $833.94 \pm 1.33$ & $2.73 \pm 0.92$ & $7.74 \pm 0.95$ & $3.31 \pm 0.50$ \\
\hline
\end{tabular}
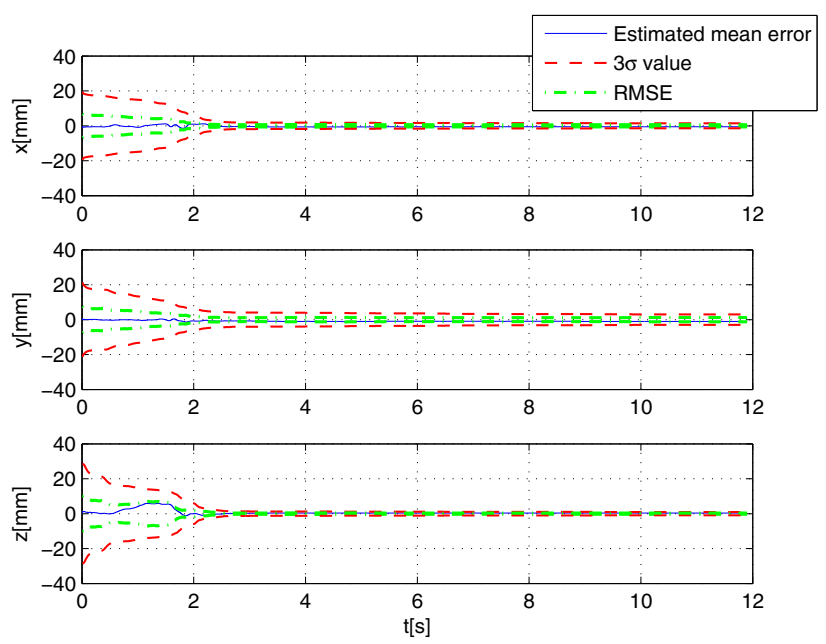

Fig. 5: Estimation error of one feature point position relative to the camera coordinate frame.
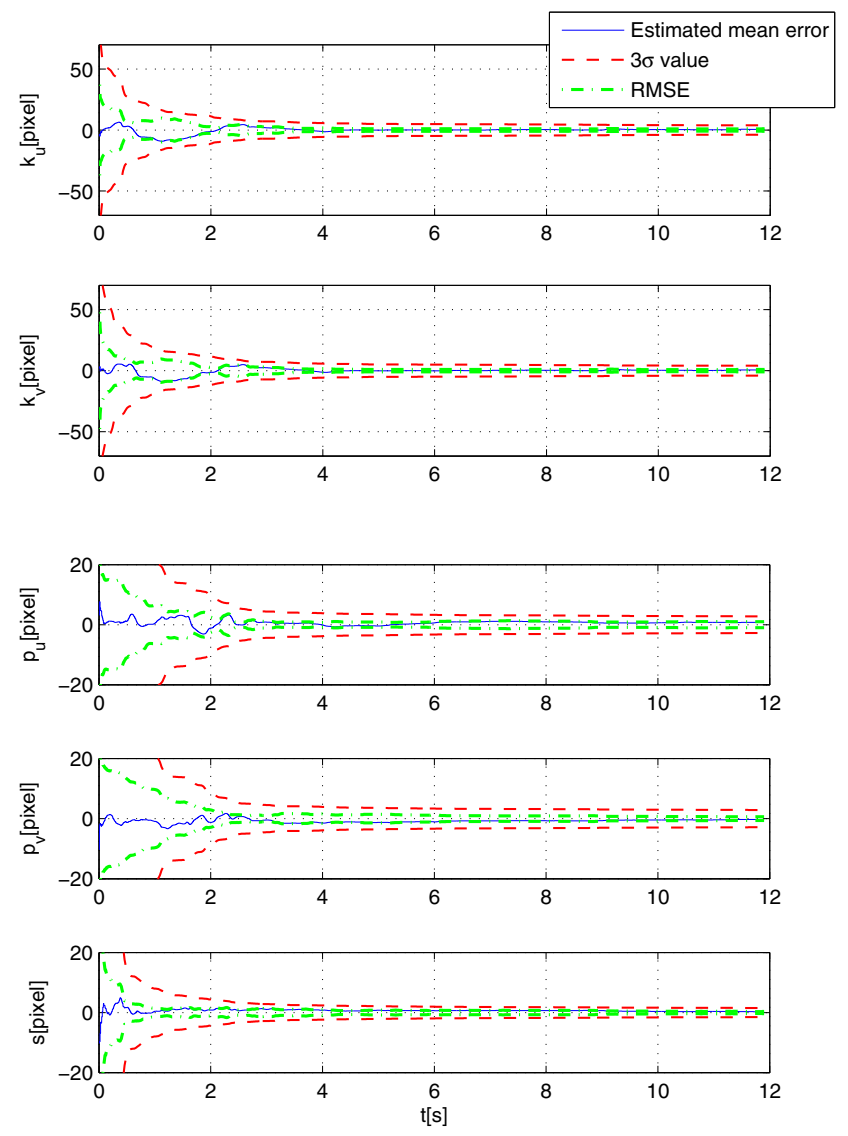

Fig. 6: Estimation error of the camera intrinsic parameters. 
tion. The method does not require a fixed calibration pattern with known feature point positions. Instead, our calibration method is based on IPMR of the feature points that are fixed in the camera body frame where no prior knowledge of their positions is assumed. Relating the IPMR with the IMU measurements, we introduced a model which has been used in the Sigma-Point Kalman filter framework to estimate the IMU-camera calibration parameters as well as the position of the feature points. Moreover, no restriction for the IMUcamera movement in front of the planar mirror is considered; however, the reflection of the feature points in the plane mirror should be visible in the images.

Additionally, using the estimated parameters, we showed that it is possible to estimate the camera intrinsic parameters in the same Sigma-Point Kalman filter approach extended with the camera calibration matrix parameters.

\section{REFERENCES}

[1] J. Lobo and J. Dias, "Relative pose calibration between visual and inertial sensor," Int. Journal of Robotics Research, vol. 26, pp. 561575, Jun. 2007.

[2] F. Mirzaei and S. Roumeliotis, "A kalman filter-based algorithm for imucamera calibration," in Proceedings of Int. Conf. on Intelligent Robots and Systems,IEEE/RSJ, pp. 2427-2434, Nov. 2007.

[3] J. Hol, T. Schön, and F. Gustafsson, "Relative pose calibration of a spherical camera and an imu," in Proceedings of 7th IEEE/ACM Int. Symposium on Mixed and Augmented Reality, ISMAR., pp. 21-24, 2008.

[4] S. J. Julier and J. K. Uhlmann, "A new extension of the kalman filter to nonlinear systems," in Proceedings of Signal Processing, Sensor fusion, and Target Recognition, vol. 4, pp. 182-193, Apr. 1997.

[5] J. Gluckman and S. Nayar, "Planar catadioptric stereo: geometry and calibration," in Proceedings of Computer Vision and Pattern Recognition. IEEE Computer Society Conference, 1999.

[6] J. Gluckman and S. K. Nayar, "Catadioptric stereo using planar mirrors," Int. J. Comput. Vision, vol. 44, pp. 65-79, Aug. 2001.

[7] J. A. Hesch, A. I. Mourikis, and S. I. Roumeliotis, "Mirror-based extrinsic camera calibration," in The Eighth Int. Workshop on the Algorithmic Foundations of Robotics, Mexico, Dec. 2008.

[8] R. I. Hartley and A. Zisserman, Multiple View Geometry in Computer Vision. Cambridge University Press, ISBN: 0521623049, 2000.

[9] R. Rodrigues, J. P. Barreto, and U. Nunes, "Camera pose estimation using images of planar mirror reflections," in Proceedings of the 11th European conf on Computer vision: Part IV, ECCV'10, pp. 382-395, Springer-Verlag, 2010.

[10] J. Y. Bouguet, "Camera calibration toolbox," Computation vision at Caltech, www.vision.caltech.edu/bouguetj/, 2008.

[11] J. M. Frahm and R. Koch, "Camera calibration with known rotation," in Proceedings of IEEE Int. Conf. Computer Vision ICCV, pp. 1418-1425, 2003.

[12] G. Panahandeh, D. Zachariah, and M. Jansson, "Mirror based imucamera and internal camera calibration," in Proceedings of IEEE Int. Conf. on Robot, Vision and Signal Processing, Nov. 2011.

[13] J. A. Farrell and M. Barth, Global Positioning System, Inertial Navigation and Integration. McGraw-Hill Companies, 1999.

[14] T. Kailath, A. Sayed, and B. Hassibi, Linear estimation. Prentice-Hall information and system sciences series, Prentice Hall, 2000.

[15] D. Zachariah and M. Jansson, "Joint calibration of an inertial measurement unit and coordinate transformation parameters using a monocular camera," in Proceedings of IPIN 2010, Sep. 2010. 